\section{Commentary: Extracorporeal membrane oxygenation cannulation for postcardiotomy shock-Is location really everything?}

\author{
Siamak Mohammadi, MD, FRCSC, and \\ Dimitri Kalavrouziotis, MD, FRCSC
}

Despite the significant advances in perioperative care and increasing cumulative experience with venoarterial (VA) extracorporeal membrane oxygenation (ECMO), the clinical outcomes associated with VA-ECMO for postcardiotomy cardiogenic shock (PCS) are still dismal. ${ }^{1,2}$ Results may be partly related to the lack of a solid literature with adequate sample sizes, which leads to a significant variability in both deployment technique and individual results, and thus inhibits us from understanding the key elements that are necessary to ensure the success of VA-ECMO for PCS. In our center and many others, VA-ECMO for PCS has gained more attention and interest in recent years because of its wide availability, flexibility of cannulation, and capacity to provide blood oxygenation relative to other temporary mechanical assist devices. The best configuration of VA-ECMO for PCS, and the factors associated with a favorable clinical outcome, however, have not been extensively investigated in a large cohort of patients.

In this issue of the Journal, Mariscalco and colleagues ${ }^{3}$ report their retrospective analysis of data from a large, multinational cohort of 781 consecutive adult patients in 19 different cardiac surgery institutions. Mariscalco and colleagues $^{3}$ performed a robust statistical analysis with inverse probability weighting by propensity score to evaluate the role of cannulation strategy (peripheral vs central) in the clinical outcomes of VA-ECMO for PCS. They concluded

From the Division of Cardiac Surgery, Quebec Heart and Lung Institute, Quebec City, Quebec, Canada.

Disclosures: Authors have nothing to disclose with regard to commercial support.

Received for publication Oct 22, 2019; revisions received Oct 22, 2019; accepted for publication Oct 23, 2019; available ahead of print Nov 13, 2019.

Address for reprints: Siamak Mohammadi, MD, FRCSC, Division of Cardiac Surgery, Quebec Heart and Lung Institute, 2725 Chemin Sainte-Foy, Quebec City, Quebec G1V 4G5, Canada (E-mail: siamak.mohammadi@fmed.ulaval.ca).

J Thorac Cardiovasc Surg 2020;160:1220-1

$0022-5223 / \$ 36.00$

Copyright (c) 2019 by The American Association for Thoracic Surgery

https://doi.org/10.1016/j.jtcvs.2019.10.132

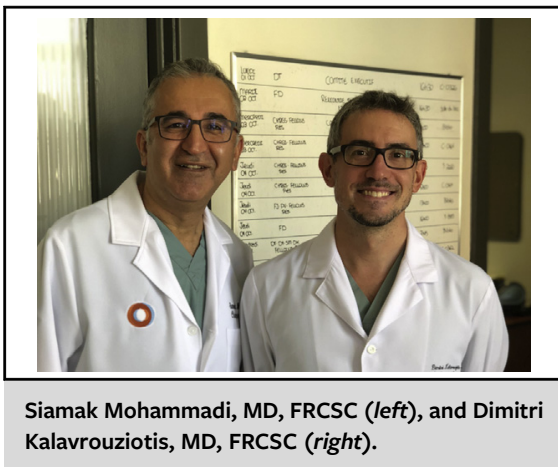

CENTRAL MESSAGE

Peripheral versus central cannu-

lation for VA-ECMO influences

outcomes in postcardiotomy

shock greatly. It remains unclear

whether this is a true cause or a

simply a surrogate of patient-

level risk.

that a central cannulation strategy was independently associated with higher in-hospital mortality $(71 \%$ vs $61 \%$ for peripheral cannulation; adjusted odds ratio, 1.5 ; $95 \%$ confidence interval, 1.1-2.2), reoperation for bleeding, and massive transfusion. Surprisingly, there were no observed differences in terms of peripheral vascular complications between the 2 cannulation strategies in both intention-totreat and as-treated analyses. They reinforced their conclusions by performing a systematic review of studies, including nearly 2500 patients with PCS and VA-ECMO, and they found that the pooled unadjusted risk ratio was lower among patients with peripheral cannulation for the end point of in-hospital or 30-day mortality and for reoperation for bleeding.

Mariscalco and colleagues ${ }^{3}$ are to be congratulated for their continuing efforts to shed some light on a field driven almost entirely by expert opinion and small, single-center observational studies by reporting the outcomes of a multicenter PCS VA-ECMO registry with a very large number of patients. The major conclusion of this article ${ }^{3}$ was that peripheral cannulation (in large part through the femoral artery and vein) was associated with better early mortality compared to central cannulation for PCS VA-ECMO. 
Mariscalco and colleagues ${ }^{3}$ suggest that this finding is due mostly to the excessively poor outcomes of a central cannulation strategy because of ongoing bleeding from the open chest and massive blood transfusion, defined as more than 9 units of red blood cells. Any attempt to impute a direct causal relationship between multiple blood transfusions and higher mortality among patients receiving central cannulation, however, remains speculative. Serious complications associated with massive red blood cell transfusion, such as transfusion-related acute lung injury and disseminated intravascular coagulation, were not reported.

Although a robust statistical analysis was performed, the conclusions should be interpreted with caution for the following reasons. First, the absence of uniform patient selection and VA-ECMO indication criteria, as well as varying postoperative management algorithms across institutions, introduces a significant source of bias that simply cannot be measured and accounted for in the multivariate propensity-score analyses. Second, the decision made to retain the central cannulas after cardiopulmonary bypass may reflect the surgeon's aversion to cannulate peripherally for a number of reasons (small peripheral vessels, extensive iliofemoral occlusive disease, advanced shock and vasoconstricted state), all of which confer an increased risk after cardiac surgery. This is corroborated by the fact that there were more patients in critical condition with a salvage indication who underwent a central cannulation strategy. In addition, the patients with central cannulation had a similar rate of lower limb ischemia to that among the patients with peripheral cannulation, which could be the result of a more advanced shock state, ${ }^{4}$ which in turn translates to a higher risk of mortality. Third, there was an extremely high overall in-hospital mortality among patients with a central cannulation strategy, exceeding $70 \%$, and there was a higher use of peripheral cannulation in higher-volume centers. The same research group has also reported ${ }^{5}$ that the volume-risk relationship is crucial for VA-ECMO, and that centers with higher experience had a $27 \%$ lower relative risk of inhospital mortality than lower-volume centers. The piece of the puzzle that is unfortunately still missing is the mechanism by which a peripheral cannulation strategy could potentially improve survival. Theoretically, peripheral VA-ECMO is associated with increased afterload, left ventricular wall tension, and myocardial oxygen consumption as a result of incomplete left ventricular unloading. A central cannulation strategy allows a higher degree of left ventricular unloading because of central venous drainage and the option for direct left ventricular emptying through a left-sided cannula; this option was used is less than onefifth of the central cannulation group for reasons that are not clear. Massive transfusion cannot be the sole explanation for the relatively poorer outcomes observed among patients with central cannulation, because almost $65 \%$ of patients with peripheral cannulation also had massive transfusion in the study of Mariscalco and colleagues. ${ }^{3}$ The answer seems to lie in patient-level factors incompletely adjusted for in the multivariate analyses, which translates to an important selection bias. Patient selection may continue to be the number one determinant of clinical outcomes in this challenging population. It is unlikely that the results of this study will significantly change individual practice. If peripheral cannulation is possible, then do it. But is it preferable at all costs to central cannulation? Probably not.

The study by Mariscalco and colleagues ${ }^{3}$ is highly nuanced and well conducted, and it is a welcome addition to a sparse literature in an area in which randomized trials are unlikely to be performed. The analysis of these data will resonate with all cardiac surgeons involved in the care of the patient with PCS, mainly because it highlights the need, with some urgency, for further collaborative and prospective research to improve the outcomes of this highly lethal condition. These types of studies are important as we gradually uncover the critical elements that will allow better clinical outcomes in VA-ECMO for PCS.

\section{References}

1. Biancari F, Perrotti A, Dalén M, Guerrieri M, Fiore A, Reichart D, et al Meta-analysis of the outcome after postcardiotomy venoarterial extracorporeal membrane oxygenation in adult patients. J Cardiothorac Vasc Anesth. 2018; 32:1175-82.

2. Rastan AJ, Dege A, Mohr M, Doll N, Falk V, Walther T, et al. Early and late outcomes of 517 consecutive adult patients treated with extracorporeal membrane oxygenation for refractory postcardiotomy cardiogenic shock. J Thorac Cardiovasc Surg. 2010;139:302-11.e1.

3. Mariscalco G, Salsano A, Fiore A, Dalén M, Ruggieri VG, Saeed D, et al. Peripheral versus central extracorporeal membrane oxygenation for postcardiotomy shock: multicenter registry, systematic review and meta-analysis. J Thorac Cardiovasc Surg. 2020;160:1207-16.e44.

4. Warkentin TE. Microvascular thrombosis and ischaemic limb losses in critically ill patients. Hamostaseologie. 2019;39:6-19.

5. Biancari F, Dalén M, Fiore A, Ruggieri VG, Saeed D, Jonsson K, et al. Multicenter study on postcardiotomy venoarterial extracorporeal membrane oxygenation. $J$ Thorac Cardiovasc Surg. July 2, 2019 [Epub ahead of print]. 